\title{
LA CAÍDA DE LA FECUNDIDAD Y LOS DERECHOS REPRODUCTIVOS EN BRASIL: UN DESAFÍO URGENTE
}

\author{
Lorena Holzmann da Silva*
}

\section{PRESENTACIÓN}

La caída de la fecundidad es uno de los fenómenos demográficos más significativos en Brasil en las últimas décadas, tanto por la rapidez como por la intensidad con que viene ocurriendo. El argumento de los riesgos de la explosión demográfica que algunos años atrás era utilizado como justificación de recomendaciones controladoras, hoy no tiene cómo sustentarse a la luz de los datos más recientes, que revelan un comportamiento demográfico que tiende a la estabilización, con tasas moderadas de natalidad y mortalidad y, consecuentemente, reducción del ritmo de crecimiento de la población.

Este comportamiento forma parte de las transformaciones estructurales de la sociedad brasileña, en el tránsito de un contexto agro-exportador hacia una sociedad urbano-industrial en corto espacio de tiempo, con todas las implicaciones económicas, sociales, políticas, culturales, de valores y comportamientos que acarreó.

Este intenso y rápido proceso de transformación ha sido excluyente y altamente concentrador de la riqueza, produciendo grandes desigualdades interregionales e intersectoriales. Los grandes contrastes son una presencia constante en la sociedad brasileña. La gran mayoría de la población vive hoy en áreas urbanas, bajo condiciones económicas, sociales y culturales de la sociedad modernizada, pero la oferta de los bienes y servicios que ella produce y la calidad de los mismos son diferentes para los distintos segmentos de la población.

En relación al comportamiento reproductivo, existe una disposición generalizada y favorable a la regulación de la fecundidad, pero el acceso a la información sobre los diferentes métodos, sus implicaciones y riesgos, las posibilidades de opción entre las diversas alternativas existentes son aún bastante precarias para la gran mayoría de la población. No hay un política explícita de planificación familiar por parte del Estado, pero en instituciones públicas de atención sanitaria se hace una orientación hacia la regulación de nacimientos y una prescripción de métodos que la viabilicen, lo que implica la ausencia de compromiso del poder público con la política que es, de hecho, practicada. En este proceso participan también instituciones privadas que desarrollan una acción complementaria a la de la esfera pública, a veces asociadas o con convenios con ésta (Ribeiro, 1993).

Traducción del portugués: Ana Inés López-Accotto.

(*) Directora del Departamento de Sociología de la Universidad Federal de Rio Grande o Sul/Brasil.
Este texto analiza las modificaciones en la fecundidad de la población brasileña, el contexto en que ocurre, los procedimientos anticonceptivos responsables de su declive, su utilización en diferentes segmentos socio-económicos de la población y lo que este proceso representa -o deja de representar- de posibilidades para las mujeres, de control de su propio cuerpo y sobre su función reproductiva y de conquista efectiva de la plena ciudadanía y de los derechos reproductivos.

\section{EL CONTEXTO BRASILEÑO DE LA CAÍDA DE LA FECUNDIDAD.}

A partir de la década de 1950, el proceso de industrialización en el país se aceleró, acompañado de un rápido e intenso proceso de urbanización que invirtió, en un espacio de 40 años, la proporción de población residente en áreas urbanas sobre la población total. En 1950, el 36,2\% de los brasileños vivían en ciudades, elevándose esta proporción al 75,5\% en 1991 (Oliveira, 1993). La migración del campo a la ciudad tiene un peso incuestionable en este proceso, integrando a la vida urbana a grandes contingentes de población que pasan a vivir nuevas relaciones sociales, económicas, políticas y culturales, incorporando nuevos hábitos y valores, redefiniendo sus referencias culturales tradicionales, construyendo nuevas expectativas de vida, sin que necesariamente las condiciones objetivas les garanticen el acceso efectivo a nuevas oportunidades de vida. Las grandes transformaciones económico-sociales que hicieran de Brasil, en la década de los ochenta, la octava economía industrial del mundo, no produjeron una distribución equitativa de los resultados de este gran crecimiento al conjunto de la población. Estilos de vida, pautas de consumo de bienes y servicios, calidad de vida similares a los del Primer Mundo conviven con una inmensa deuda social, caras opuestas de una misma moneda, del mismo proceso de modernización acelerada que transforma a toda la sociedad, provocando cambios profundos también en el comportamiento de las personas. Una de las expresiones de estos cambios se traduce en el comportamiento reproductivo y en las actitudes relacionadas con él.

Es a partir de los años 60 cuando se inicia un persistente proceso de desaceleración del crecimiento demográfico. Entre 1920 y 1960 la población brasileña creció de 27, 5 millones a 70,1 millones, debido exclusivamente al crecimiento vegetativo, ya que la inmigración no tuvo una contribución significativa en este incremento. En la última década de aquel período el aumen- 
to de la población presentó una tasa de 3,17\% anual, alimentando los argumentos de los heraldos de la explosión demográfica. Este fenómeno no fue exclusivo de Brasil, ya que una elevación en las tasas de crecimiento de la población también se verificó en los países desarrollados, en los cuales aquel crecimiento ya había alcanzado, con anterioridad, una relativa estabilidad. Pero en éstos, el crecimiento de la población en el período fue el resultado de una elevación puntual en las tasas de fecundidad, en tanto que en Brasil se debía al mantenimiento de niveles elevados de fecundidad y de niveles tendencialmente declinantes de mortalidad, aunque ésta se mantenía en niveles elevados. Pero a partir de la década de los 60 este cuadro se altera, cayendo progresivamente en las décadas siguientes. Entre 1960 y 1970 el crecimiento anual de la población fue del 2,76\%, entre 1970 y 1980 , el $2,48 \%$ y entre 1980 y 1991 , el $1,89 \%$.

La inflexión en la tasa de crecimiento de la población que se da a partir de la década de los 60, resulta particularmente de la caída acelerada de la tasa de fecundidad. En el decenio 1950-60 la fecundidad total de las mujeres brasileñas era de 6,3 hijos, media que fue bajando continuamente (5,8 entre 1960-70; 4,8 entre 1970-80), hasta alcanzar 2,7 hijos por mujer entre $1980 \mathrm{y}$ 1991. Estas medias presentan diferencias significativas entre las regiones del país, registrándose la media más elevada entre 1950-60 en la región Norte, con 8,6 hijos por mujer y la más baja, de 5,9, en la región Sur. En 1991 esta región continúa con una media más baja (2,3 hijos por mujer) y las regiones Norte y Nordeste con la más elevada, de 4 hijos por mujer (Oliveira, 1993). La fecundidad es más elevada en las regiones más atrasadas y esta relación se mantiene a lo largo del tiempo, confirmando la relación inversa, históricamente recurrente, entre fecundidad y desarrollo económico-social.

La diferencias de fecundidad están asociadas a otras variables, como escolaridad y rentas, observándose la disminución del número de hijos con el aumento de esos indicadores, con diferencias también significativas si estos subconjuntos fueran considerados de acuerdo con el lugar de residencia: las mujeres de áreas rurales tienen más hijos que las habitantes de áreas urbanas (Simoes y Oliveira, 1988).

A pesar de todas estas diferencias, es común a todos los subconjuntos de mujeres la tendencia a la reducción de la fecundidad, evidenciando la difusión, en todos los estratos de la población, de prácticas de regulación de los nacimientos.

La tendencia recurrente de la caída de la fecundidad en sociedades en desarrollo presenta diferencias en cuanto a la intensidad y a la rapidez con que ocurre, siendo decisivas tanto la forma como la mujer se inserte en las transformaciones estructurales producidas y el papel que le es reservado o atribuído en las estrategias de desarrollo (Young, 1989), como de la presencia o no de una serie de otros factores tales como la existencia de políticas públicas de planeamiento familiar (Kabeer, 1992), cambios en el status social y cultural de las mujeres, su ingreso en el mercado de trabajo, la difusión y aceptación de orientación favorable a la regulación de la fecundidad y el acceso a métodos que la hagan efectiva, la elevación en el nivel de escolaridad general y sus repercusiones en el interior de la familia, entre otros. Estos factores son relevantes en los cambios de comportamiento reproductivo. Por ejemplo, la entrada en el mercado de trabajo, tanto por razones predominantemente económicas tales como la necesidad de supervivencia o expectativas de expansión del consumo (Singer, 1977), así como en el transcurso de transformaciones culturales que rompen con la estrategia de los papeles tradicionalmente atribuidos a la mujer y abren perpectivas de incorporación de un proyecto profesional como parte de un proyecto de vida, no puede ser ignorada; las dificultades de conciliar las cargas profesionales con las cargas familiares de una prole numerosa actúan en el sentido de favorecer la emergencia de una disposición favorable a la regulación de los nacimientos y a la búsqueda de procedimientos que la hagan efectiva.

Algunos de estos factores estuvieron presentes en el contexto brasileño. En 1980, la proporción de mujeres con 10 años de integración en la población económicamente activa era del 26,6\%, elevándose al 39,2\% en 1990 (CUT/DESEP, 1994), incrementando la participación femenina en la población económicamente activa del $31,2 \%$ al 35,6\%. La proporción de familias brasileñas en que el cabeza de familia y su cónyuge trabajaban se elevó del $13,6 \%$ en 1981 al $18,1 \%$ en 1987 y del $5,8 \%$ al $7 \%$ la proporción de familias en las que el cabeza de familia, cónyuge y uno o más hijos estaban en el mercado de trabajo. Estos datos excluyen a las familias monoparentales encabezadas y sustentadas por mujeres que en el mismo período aumentaron del 10,2\% al 11,9\% (Castelo Branco, 1989). La modernización de la sociedad brasileña explica, en parte, la entrada de la mujer en el mercado de trabajo, así como su carácter concentrador y excluyente ha provocado la entrada de menores en el mercado de trabajo, en condiciones legales y de remuneración muy precarias. En 1990, el $17,2 \%$ de los niños de 10 a 14 años y el $50 \%$ de los adolescentes de 15 a 17 años trabajaban, de los cuales el 86,2\% recibían una remuneración inferior a un salario mínimo y el 74,4\% no tenían contrato de trabajo firmado (Oliveira, 1993), lo que los excluye del acceso a los beneficios y garantías de la legislación laboral. Estos factores indiscutiblemente tienen implaciones en el seno de la familia, siendo los cambios en la fecundidad uno de ellos.

Considerando insatisfactorias las explicaciones macroestructurales que consideran la caída de la fecundidad como un resultado debido básicamente al proceso de modernización/ urbanización/industrialización, Farias (1989) busca identificar, en el caso de Brasil, la contribución de otros factores en la caída de las tasas de fecundidad ocurrida en las últimas décadas, privilegiando en su análisis políticas gubernamentales implantadas a partir de los años 60. El autor argumenta que aunque éstas no tuviesen como objetivo alterar el comportamiento reproductivo de la población, tuvieron efectos relevantes sobre éste. Expuestas de modo resumido, estas políticas son:

a) La política de crédito al consumidor que habría reforzado el cálculo económico en la vida de los individuos, ya sometidos a un patrón de vida mercantilizado debido a su condición de asalariados en la economía urbana. Fue esta política la que permitió que, aún en un proceo de gran concentración de la renta, amplios segmentos de la población fueron teniendo acceso a bienes duraderos de consumo, de precios elevados. Así, en 1990, el 71,1\% de los domicilios estaban equipados con neveras, el $73,7 \%$ con aparatos de televisión y el $84,3 \%$ con radios (DIEESE, 1993) elevándose esta proporción al $71,7 \%$ (neveras), $75,8 \%$ (televisores) y $85,1 \%$ (radio) en 1993 (IBGE, 1996)

El acceso a estos bienes, facilitado por la política de crédito, no significó necesariamente mejoras en la calidad de vida, como revelan los datos relativos a las condiciones sanitarias 
de los domicilios en el país en 1988, de los cuales apenas el $54,6 \%$ tenían redes de desagüe, el $60,1 \%$ contaban con servicio de recolección de basura y el 70,9\% eran abastecidos de agua canalizada a través de red centralizada (IBGE, 1990).

b) La política de expansión de las telecomunicaciones en particular de la televisión y su papel decisivo en la formación de la opinión pública y en la transmisión de patrones de comportamiento, hábitos y valores "modernos", entre ellos la disociación de sexualidad y reproducción.

c) La política de expansión de los beneficios previsionales, ampliando de modo significativo la población atendida. Al mismo tiempo fueron debilitados los mecanismos tradicionales de apoyo, comunitarios y de parentesco, para enfrentar situaciones de adversidad, como enfermedades, accidentes, desempleo, viudez, con implicaciones sobre el papel y las expectativas que los grupos primarios desempeñaban hasta entonces.

d) La política de atención a la salud, que llevó a una creciente medicalización de la sociedad bajo la autoridad del saber médico, en detrimento de prescripciones empíricas asentadas en el saber popular, respaldando y sancionando actitudes y prácticas de regulación de la fecundidad.

Estas políticas formuladas e implementadas durante el régimen militar, buscaban tanto legitimarlo delante de la población (expansión del sistema previsional y de atención a la salud) cuanto garantizar medios más eficaces de control (telecomunicaciones) y sustentación de un nuevo patrón de acumulación de capital (política de crédito). Las consecuencias de estas acciones gubernamentales,según el autor, no anticipadas, se combinaron para acelerar procesos de cambio sociocultural en el sentido de una disposición a la regulación de la fecundidad en todos los segmentos de la población.

Esta disposición se hace efectiva con la utilización de procedimientos anticonceptivos, haciendo caer rápidamente la tasa de fecundidad.

Las condiciones en que estos procedimientos son utilizados dicen mucho al respecto de la mala calidad de la salud reproductiva de las mujeres brasileñas y de la precaria vigencia de opciones efectivas de contracepción, dejando mucho que desear en términos de derechos reproductivos.

\section{PRÁCTICAS ANTICONCEPTIVAS EN BRASIL}

La PNAD (Encuesta Nacional de Viviendas) realizada en 1986 y publicada en 1991 es la fuente más completa y amplia sobre anticoncepción en el país, englobando todo el territorio nacional y todos los segmentos de la población. Desgraciadamente es el estudio más reciente con este nivel de amplitud. Se han realizado otras investigaciones, pero aunque su contribución para el conocimiento más preciso y detallado de estas cuestiones relacionadas con las prácticas anticonceptivas no puede ser ignorado, son, en general, estudios de caso, con alcance limitado.

La PNAD investigó el conjunto de la población femenina en la franja de edad de 15 a 54 años, con un total de 37.318 .709 mujeres. De este total, $22 \%$ eran usuarias de métodos anticonceptivos (MAC) lo que representaba 8.211,957 mujeres.

La encuesta no recogió datos respecto de la interrupción deliberada del embarazo como procedimiento regulador del tamaño de la prole. El aborto es ilegal en Brasil, pero es amplia- mente practicado clandestinamente, tanto en clínicas médicas particulares como por personas legas, sin ninguna cualificación para este tipo de intervención, implicando grandes riesgos y provocando una alta incidencia de mortalidad y morbilidad entre las mujeres, particularmente las de baja condición socio-económica. Estas recurren a legos, pagando por el aborto mucho menos de lo que se cobra en las clínicas clandestinas, cuyos precios sólo pueden ser pagados por mujeres de mejor situación económica, con garantías de mayor seguridad, inexistentes para las mujeres pobres. Estas cuestiones han sido planteadas en el debate sobre el aborto, suscitando el argumento, entre los que defienden su despenalización, de que su ilegalidad, además de ser hipócrita, por no encarar un problema existente y grave, es discriminatoria, penalizando en especial a las mujeres pobres que no pueden recurrir a éste con seguridad y pocos riesgos, como lo hacen las otras mujeres. Estas, aunque puedan pagar mucho por la intervención y realizarla con menos riesgos, quedan sujetas a la extorsión de los profesionales que actúan en la clandestinidad, sin estar sujetos a ningún control o fiscalización de los servicios de salud. Además de eso, no es posible ignorar el gran número de abortos practicados en el país, estimado entre 1 y 4 millones por año (Barbosa y Arilha, 1993) y su lugar entre las cuatro causas principales de mortalidad materna (Tanaka, 1994)

La encuesta del PNAD registró como no usuarias de MAC el significativo número de 5.900.238 mujeres que habían sido esterilizadas y que respresentaban el $15,8 \%$ de las mujeres en la franja de edad considerada. Volveremos a la consideración de este hecho más adelante, pues merece un examen más detallado. Por ahora vamos a tratar las condiciones de las mujeres usuarias de MAC.

En relación al lugar de residencia, no hay diferencias muy significativas en la utilización de MAC entre habitantes de zonas urbanas y de zonas rurales; estre éstas la proporción de usuarias era de $19,4 \%$ y entre aquéllas de $22,8 \%$. Este dato revela que los cambios socioculturales en el sentido de regular la fecundidad se difunden en todos los segmentos de la población, penetrando también entre los más tradicionales. En cambio, en lo que se refiere a las condiciones de seguimiento y consejo médico requerido en la utilización de métodos anticonceptivos, en especial los orales y los dispositivos intra-uterinos, necesarios para preservar la salud de la mujer y garantizarle comodidad, seguridad y bienestar, la situación era bastante desigual entre las mujeres residentes en zonas urbanas o rurales. Entre las que vivían en ciudades, el 14,2\% de las usuarias de MAC no tenían ningún tipo de seguimiento, mientras que entre las que vivían en el medio rural esta proporción se elevaba al $34,5 \%$. Para el conjunto de las mujeres de la franja de edad considerada la proporción de atención a la salud era significativamente inferior, pues entre las habitantes de zonas urbanas el 31,1\% no tenían ningún seguimiento de salud, llegando esta proporción al $51,6 \%$ entre las mujeres de las zonas rurales. Se verifica, por estos datos, que hay una disposición mayor a un seguimiento de salud entre mujeres usuarias de MAC y que los cuidados con la salud están estrechamente vinculados con su función reproductiva, relegando al plano secundario procedimientos preventivos de alcance más integral.

La degradación de los servicios públicos de salud, su oferta desigual para las poblaciones, urbanas y rurales, dificulando más a éstas que a aquéllas el acceso efectivo a los mismos son algunos de los factores que dan cuenta de la persistencia de esas 
diferencias. Se supone que otros factores como la propia intensidad diferencial del alcance del proceso de medicalización de la sociedad (Farias, 1989) pueden explicar las diferencias existentes, mas los datos en que se basó este trabajo no permiten aprehender los matices de este proceso.

$\mathrm{Al}$ considerar la frecuencia de utilización de MAC en las diferentes cohortes, se verifica que es en el grupo de 25 a 34 años donde se encuentra la mayor proporción de usuarias $(33,8 \%)$, seguido por el grupo de $35-44$ años, con $23,8 \%$. Entre el grupo de 45 a 54 años se reduce considerablemente la proporción de usuarias, apenas el $8,7 \%$.

En los grupos de edad más jóvenes es significativa la proporción de usuarias de MAC. El 27,4\% entre las mujeres con edades de 20 a 24 años, que se reduce significativamente entre las que tienen de 15 a 19 años $(7,8 \%)$. La baja proporción de mujerers en esta última cohorte que son usuarias de MAC coexiste con el aumento de la participación de la misma en la fecundidad total registrada entre 1970 y 1984. En aquel año, niñas de 15 a 19 años contribuían con el 6,5\% en la fecundidad total, elevándose esta a 9,1\% en 1980 y al 10,5\% en 1984 (Simoes e Oliveira), 1988). Estos datos evidencian el preocupante aumento de la incidencia de embarazos en la adolescencia.

Una vez introducidas estas informaciones más generales que dan cuentra de la variación de la frecuencia en la utilización de MAC, pasamos a examinar como se comporta la población femenina brasileña en cuanto a la elección de los métodos y por qué motivos la hacen.

\section{MÉTODOS ANTICONCEPTIVOS: ¿HAY LIBERTAD DE ELECCIÓN?}

Las usuarias de MAC en 1986 en Brasil eran 8.211.957 mujeres, de las cuales el 73,4\% daban preferencia a los anticonceptivos orales. El segundo procedimiento más utilizado era la "tabela" (referencia popular al método de Ogino-Knaus), declarado por el $11,5 \%$ de las mujeres, seguido por el coitus interruptus $(5,3 \%)$ y por el uso del preservativo $(3,7 \%)$. Se puede suponer que en relación a este método ha habido alguna alteración en años recientes como consecuencia de las campañas de prevención del sida. Estas, sin embargo, han enfatizado el uso del preservativo como recurso contra la diseminación de la enfermedad, dándose poca importancia a su posibilidad como método anticonceptivo. La inexistencia de datos más recientes e insuficientemente amplios para llegar a conclusiones más categóricas, no permite evaluar si hubo cambios cuantitativos relevantes en la utilización de este método, ni verificar si su uso ha servido también a la anticoncepción o si está restringido a la prevención del contagio de HIV.

El DIU -dispositivo intrauterino- es utilizado por apenas el $2,3 \%$ de las mujeres.

Sumados, estos cinco métodos representan el $96 \%$ de los procedimientos adoptados por las mujeres brasileñas, con una evidente preferencia por la píldora.

La gran proporción de usuarias de este método trae a la discusión el predominio del papel de la mujer en la práctica y en la responsabilidad de la anticoncepción, reforzada por la orientación de la investigación en la búsqueda de perfeccionamiento de modernos MAC. Kabeer menciona una estimación según la cual apenas el $8 \%$ de los gastos de investigación en anticoncepción en el mundo tiene como objetivo el desarrollo de métodos de uso masculino y llama la atención sobre el papel secundario que los hombres tienen en los programas y grupos que se ocupan de la planificación familiar, definiendo a los hombres como "el género invisible" en este proceso (Kabeer, 1992: 20-18).

En Brasil las condiciones no son diferentes. Los métodos que requieren alguna forma de colaboración del compañero (preservativo, interrupción del acto, abstinencia, Ogino, billing) son utilizados por apenas el $21,5 \%$ de las mujeres, evidenciando que en la gran mayoría de los casos recae exclusivamente sobre las mujeres la responsabilidad, el peso y los riesgos de la anticoncepción. Los procedimientos a los cuales están más frecuentemente asociados efectos colaterales negativos, en especial los anticonceptivos orales y el DIU, eximen al compañero de cualquier implicación, mientras que los que suponen esta implicación requieren apenas disposición a cooperar, sin exponer al cuerpo a intervenciones químicas, hormonales o mecánicas permanentes y a los riesgos de los mismos.

La participación de los hombres brasileños en las cuestiones anticonceptivas es inferior a la media del conjunto de los países en desarrollo, donde fue registrada una proporción aproximada del 25\% en la década de los 70 e inicio de los años 80 (Kabeer, 1992: 19).

Si tomamos en consideración, en el caso de Brasil, el elevado número de esterilizaciones quirúrgicas realizadas a mujeres e incluimos a estas mujeres en el grupo de las que usan algún procedimiento anticonceptivo, la participación masculina en la anticoncepción quedará aún más reducida $(12,5 \%)$. Un dato relevante y que refuerza este dato es lo relativo del número de mujeres que no utilizaban ningún MAC porque sus compañeros habían sido esterilizados: 168.219 mujeres, representando apenas el $0,6 \%$ del total.

La píldora es el MAC más utilizado por las mujeres brasileñas, como ya se demostró y no hay diferencias significativas en los distintos segmentos de la población femenina en cuanto a la proporción de la usuarias. Es utilizada por el $72 \%$ de las mujeres blancas y por el $77,5 \%$ de las mujeres no blancas, por el $72 \%$ de las económicamente activas y por el 74,6\% de las no económicamente activas. La gran mayoría de las usuarias de anticonceptivos orales estaba en la franja de edad de los 20 a los 34 años $(70,6 \%)$ y el $74,5 \%$ pertenecía a familias en las que el ingreso mensual medio per cápita era de dos salarios mínimos o menos (en abril de 1996, el salario mínimo era de 100 reales, equivalente, aproximadamente, a 103 dólares) y el 50,6\% tenía hasta 4 años de escolarización.

No hay exigencia de prescripción médica para la adquisición de anticonceptivos orales, que se pueden comprar en cualquier farmacia. Así, si hay motivos para un cambio de marca, lo que suele ocurrir a causa de efectos colaterales negativos, las usuarias pueden hacerlo sin ningún impedimento, así como pueden requerir el consejo de los dependientes de los establecimientos comerciales en donde son vendidos, lo que puede acarrear graves riesgos, ya que estos profesionales no tienen ninguna cualificación para la prescripción de medicamenteos, a pesar de que comúnmente lo hagan. Entre las usuarias de píldora, el 20,7\% no tienen ninguna recomendación para su uso.

Los diferentes tipos de píldoras pueden ser utilizados sin tener en cuenta las condiciones específicas de salud de cada mujer, así como pueden ser cambiados sin ninguna orientación. La posibilidad de búsqueda de un anticonceptivo oral "ideal" puede darse por la vía de un procedimiento de ensayo/error, 
según el criterio de la propia usuaria. El aumento de los riesgos que de ello deriva es innegable.

Aún cuando el uso de la píldora es prescrito (y éste es el caso del 63,6\% de las usuarias), no hay garantía de que la salud de la mujer sea el factor decisivo para orientar la prescripción. Una investigación llevada a cabo en una clínica de planificación familiar en una ciudad del interior de Sao Paulo, realizada por convenio entre el Ayuntamiento y la BENFAM, institución privada ligada a la International Planned Parendhood Federation, constató que el tipo de píldora recomendada no atendía a las peculiaridades individuales de las usuarias y sí a las marcas disponibles en la clínica y que apenas el $5 \%$ de las mujeres a quienes había sido recomendada la píldora se habían sometido previamente a una evaluación clínica y a exámenes preventivos de cáncer (Scavone, Bretin y Thébaud-Monty, 1994). La clientela de la clínica está formada por mujeres de baja renta y escolarización y una gran mayoría recurren a ella con la finalidad exclusiva de obtener consejo en planificación familiar. De las clientes entrevistadas en la investigación, el 95\% utilizaba la píldora, llevando a las autoras a la conclusión de que

El funcionamiento de este puesto evidencia una oferta contraconceptiva dirigida a las mujeres sin recursos, caracterizándose por la falta de exámenes, de indicaciones médicas y la ausencia de elección. En tal contexto, las píldoras menos dosificadas, DIUs y seguimiento médico se convierten en un verdadero "privilegio" de clase. (p.369)

En el contexto brasileño, donde los servicios de salud para la gran masa de la población son muy precarios, nada nos estimula a suponer que este caso sea una excepción.

El uso masivo de la píldora puede ser atribuido a las dificultades de acceso efectivo a otras opciones, ya que apenas el $6,1 \%$ de las usuarias declaró, en la PNAD, desconocer otro método.

Pero aun si otros procedimientos anticonceptivos son conocidos por la gran mayoría de las mujeres, es muy alta la proporción de las que no saben dónde y cómo obtenerlos (Berquó, 1993). Estas dificultades no existen con la píldora, disponible en cualquier farmacia y el método por excelencia prescrito en las instituciones de atención a la salud. La forma indiscriminada de su uso, las escasas posibilidades de corregir los efectos colaterales negativos con la sustitución por un producto más adecuado a las condiciones de cada mujer (Scavone, Bretin y Thébaud-Mony, 1994) y el desconocimiento del acceso a otros procedimientos llevó a producir, en Brasil, una "cultura de la esterilización". (Berquó, 1993).

Este procedimiento pasa a ser entendido como la solución anticonceptiva ideal, ya llevado a cabo por un número grande de mujeres y pretendido por otras tantas, inclusive por mujeres muy jóvenes (Berquó, 1993). Con esta perspectiva, es bastante razo- nable suponer que la tendencia es el aumento de la incidencia de esterilizaciones quirúrgicas entre las mujeres brasileñas, superando la proporción del 15,6\% registrada en 1986.

Este dato se refiere al conjunto de las mujeres con edades de 15 a 54 años. Si se considera sólo la población femenina de esta franja de edad en condiciones de unión y no usuaria de MAC (que totalizan 16.374 .000 mujeres), la proporción de esterilizaciones se eleva al 36\%, siendo mucho más alta entre las residentes en áreas urbanas $(40,9 \%)$ que entre las mujeres que viven en áreas rurales, donde la proporción es del $22,8 \%$.

El debate sobre este procedimiento y su difusión en Brasil ha estado marcado por controversias, entre ellas cuál es el segmento de población que más recurre a él, polarizándose el mismo en torno a una posición que apunta a las mujeres de mejor situación socio-económica y educacional como las mayores usuarias de la esterilización quirúrgica, enfrentada con otra, que denuncia este procedimiento como dirigido en especial para controlar la fecundidad de las mujeres pobres y como una práctica que atiende a los intereses de los gobiernos de los países desarrollados, viabilizados a través de agencias internacionales privadas, ejecutoras de programas de control en los países del Tercer Mundo (Terra, 1991)

Estas tesis (neo)malthusianas son rescatadas con frecuencia, asociándose el atraso o la lentitud del desarrollo al rápido crecimiento de la población, apuntando a la urgencia de medidas que lleven a la reducción de este crecimiento. Incluso en Brasil, donde la caída del ritmo de crecimiento ya se viene dando hace más de 30 años, se continúa haciendo esa asociación. La persistencia de este discurso contribuye al enmascaramiento de las causas reales de la pobreza, enraizadas en las graves distorsiones económicas y sociales vigentes en el país. La consecuencia es denunciada como causa y los pobres son responsabilizados por la pobreza porque se reproducen a través de proles numerosas. La solución, entonces, es controlar la fecundidad de las mujeres pobres.

A partir de esta lógica, que encuentra resonancia también entre la comunidad médica, la urgencia de esta solución se impone debido a la gravedad del problema y la manera más rápida y eficaz es la esterilización.

El análisis de la población femenina esterilizada, comparada con la usuaria de anticonceptivos orales, combinada con indicadores socio-económicos, permite verificar las diferencias entre mujeres de distintas condiciones socio-económicas, en la adopción de los dos MAC más utilizados en el país, así como examinar si la regulación de la fecundidad de las mujeres pobres a través de la esterilización se diferencia del mismo procedimiento adoptado por otras mujeres.

Como se observa por el examen de la tabla I, hay una relación inversa entre escolaridad y esterilización.

Entre las mujeres sin instrucción y con hasta cuatro años de

\section{Tabla I}

Procedimiento anticonceptivo, de mujeres con 15 a 54 años, según la escolarización (en años de estudio) - Brasil, 1986 (\%)

\begin{tabular}{|c|c|c|c|c|}
\hline \multirow[b]{2}{*}{ proced.anticonceptivo } & \multirow[b]{2}{*}{ sin instrucción } & \multicolumn{3}{|c|}{ años de estudio } \\
\hline & & 1-4 años & 5-7 años & + de 8 años \\
\hline píldora & 9,8 & 17,1 & 17,7 & 17,6 \\
\hline esterilización & 14,8 & 18,6 & 13,0 & 14,3 \\
\hline otro/no usan & 75,4 & 64,3 & 69,3 & 68,1 \\
\hline total & 100,0 & 100,0 & 100,0 & 100,0 \\
\hline
\end{tabular}


estudios, la proporción de esterilizadas es mayor que la proporción de usuarias de píldora, siendo la diferencia a favor de la primera mayor entre las mujeres sin ninguna instrucción que entre las que tienen hasta 4 años de escolaridad. Entre estas, la proporción de esterilizadas $(18,6 \%)$ es apenas un poco más elevada que la de usuarias de píldora $(17,1 \%)$. Aumentando la escolaridad, esta relación se invierte, siendo mayor la proporción de las usuarias de anticonceptivos orales que la de esterilizadas. Nótese, al mismo tiempo, que entre las mujeres con 8 años o más de estudio, la proporción de esterilizadas $(14,3 \%)$ es mayor que entre las mujeres que tienen entre 5 y 7 años de escolarización (13\%)

Entre las usuarias de la píldora, el 9,9\% no tiene ninguna escolarización, como se puede verificar en la Tabla II. Si sumamos a éstas las que tienen hasta 4 años de estudios, totalizaremos el 50,5\%. Entre las esterilizadas estas proporciones son respectivamente del $15,1 \%$ y del $60,3 \%$. Las mujeres con escolaridad más elevada ( 8 años o más de estudio) representan el $31,6 \%$ de las usuarias de anticonceptivos orales y $26,2 \%$ de las esterilizadas.

La exposición de estos datos permite concluir que aunque la este-

Tabla II

Escolaridad de mujeres con 15 a 54 años, según el procedimiento anticonceptivo Brasil, $1986(\%)$

\begin{tabular}{|c|c|c|c|c|c|}
\hline \multirow[b]{2}{*}{ MAC. } & \multirow[b]{2}{*}{ sin instr. } & \multicolumn{3}{|c|}{ años de estudio } & \multirow[b]{2}{*}{ Total } \\
\hline & & 1-4 años & $5-7$ años & + de 8 años & \\
\hline píldora & 9,9 & 40,6 & 17,8 & 33,66 & 100 \\
\hline esterilización & 15,1 & 45,2 & 13,4 & 26,2 & 100 \\
\hline
\end{tabular}

Fuente de los datos brutos: IBGE/PNAD, 1986

rilización sea una práctica adoptada por mujeres de diferentes niveles de escolaridad, es más frecuente entre las que tienen baja escolaridad, estrato donde es el método predominante. Obsérvase también que la proporción de mujeres con baja escolaridad entre las esterilizadas es mayor que entre las usuarias de píldoras, reconociéndose, en cambio, que la esterilización quirúrgica es una práctica con significativa aceptación entre el grupo con mayor escolarización.

Una tendencia similiar se da en relación con el nivel socio-económico, como revela la Tabla III.

Donde los ingresos familiares son más bajos (hasta un sala-

Tabla III

Procedimiento anticonceptivo de mujeres de 15 a 54 años, según el ingreso mensual familiar per cápita (en salarios mínimos) - Brasil, 1986 (\%)

\begin{tabular}{|c|c|c|c|c|c|}
\hline \multirow[b]{2}{*}{ MAC. } & \multicolumn{5}{|c|}{ salarios mínimos } \\
\hline & hasta $1 / 4$ & $1 / 4-1 / 2$ & $1 / 2-1$ & $1-2$ & $+\operatorname{de} 2$ \\
\hline píldora & 10,7 & 14,7 & 18,2 & 17,5 & 25,6 \\
\hline esterilización & 13,1 & 15,4 & 16,6 & 16,2 & 17,7 \\
\hline otros/sin & 76,2 & 70,9 & 65,2 & 66,3 & 56,7 \\
\hline Total & 100 & 100 & 100 & 100 & 100 \\
\hline
\end{tabular}

Fuente de los datos brutos: IBGE/PNAD

rio mínimo), la esterilización es el procedimiento anticonceptivo dominante, seguido por la píldora en la medida en que mejora el nivel económico de la familia. Pero se observa también que con el nivel socio-económico de la familia, aumenta la proporción de mujeres esterilizadas lo mismo que ocurre en relación a la píldora. Esto se debe a que, con la mayor escolarización, disminuye la proporción de mujeres que no utilizan procedimientos anticonceptivos y/o utilizan otros métodos. En cambio, en la clase de mayor ingreso (más de dos salarios mínimos) la proporción de usuarias de píldora es de 7,9 puntos porcentuales superior a la proporción de mujeres esterilizadas, diferencia que no se registra en ninguna de las otras clases de ingreso.

Al considerarse la condición económica de la familia, según el procedimiento anticonceptivo que adoptan, registrada en la Tabla IV, se verifica un dato significativo: la proporción de mujeres con rendimiento familiar más alto (por encima de dos salarios mínimos) es levemente más alta entre las esterilizadas que entre las usuarias de anticonceptivos orales. Este dato no invalida la tendencia a la relación inversa entre condición económica y esterilización, pero sugiere la oportunidad de una investigación cualitativa más amplia que los estudios de caso realizados, capaz de elucidar las condiciones en que mujeres de diferentes niveles socio-económicos optan y se someten a la esterilización quirúrgica.

El tema de la esterilización de mujeres en Brasil trae a cola- 
Tabla IV

Mujeres de 15 a 54 años, por ingreso familiar mensual per cápita (en salarios mínimos) según el procedimiento anticonceptivo (\%) - Brasil, 1986

\begin{tabular}{|c|c|c|c|c|c|c|}
\hline \multirow[b]{2}{*}{ MAC. } & \multicolumn{6}{|c|}{ salarios mínimos } \\
\hline & hasta $1 / 4$ & $1 / 4-1 / 2$ & $1 / 2-1$ & $1-2$ & $+\operatorname{de} 2$ & Total \\
\hline píldora & 6,8 & 15,3 & 27,1 & 25,2 & 22,6 & 100 \\
\hline esterilización & 8,5 & 16,3 & 25,1 & 23,7 & 24,7 & 100 \\
\hline
\end{tabular}

Fuente de los datos brutos: IBGE/PNAD, 1986

ción el uso electoral que políticos inescrupulosos han hecho de ella. En períodos de campaña electoral, cirugías de ligadura son ofrecidas y practicadas a cambio de votos, denunciándose la forma indiscriminada con que son hechas, atendiendo sólo a las cuentas electorales de sus patrocinadores (Istoé, 1993). Es cierto que, con mucha frecuencia, las propias mujeres la desearon y reivindicaron. Esta actitud está asociada a la dificultad de acceso a otros métodos, aunque las mujeres tengan informaciones sobre ellos. (Berquó, 1993), a la falta de estas informaciones; o la inadaptación de otros métodos, llevando a las mujeres a buscar la esterilización como una solución definitiva que las libre del riesgo de un embarazo no deseado.

Esto es evidente cuando se constata que el 59,5\% de las mujeres esterilizadas pagaron por la intervención quirúrgica, en su gran mayoría realizada en hospitales o clínicas particulares $(83,4 \%)$ y sólo una pequeña proporción en unidades de salud pública o concertadas $(13,7 \%)$. Las mujeres que no pagaron por la operación, el 49,4\% fueron esterilizadas en unidades públicas de atención de salud y 43,3\% en instituciones privadas.

Es oportuno recordar que este tipo de intervención quirúrgica está prohibida por el código penal brasileño.

Es en la condición de gratuidad de la realización de la cirugía esterilizadora donde más fácilmente se da la actuación de políticos y médicos inescrupulosos ("el doctor reconoce que en los períodos de campaña electoral hace ligaduras a petición de los políticos de la ciudad, confesando que son mis amigos, ellos me pagan, yo hago") (Istoé, 1993: 73), para quienes las intervenciones realizadas apenas tienen el carácter de un trueque clientelístico del que son intermediarios, descomprometidos con la historia de vida de las mujeres que se someten a ellas y con el respeto que les es debido.

No se trata, a priori, de condenar el procedimiento en sí, sino la forma en que viene siendo practicado en el país, según Berquó, “...es la masificación de ese procedimiento, donde el grueso de la población empeña el futuro con enorme desconocimiento de su significado, lo que constituye un grave problema de moral pública, de moral de Estado, de moral política. Un caso dramático de negación de libertad. La esterilización es el método a través del cual el control institucional deliberado puede alcanzar un grado perfecto y total de autoritarismo." (Berquó, 1982: 49)

No siempre las mujeres sometidas a la esterilización quirúrgica son informadas de que se trata de una intervención irreversible y sólo descubren este hecho cuando, reconsiderando la decisión anterior, deciden tener un hijo (IstoÉ, 1993). Es exactamente en la ausencia de informaciones precisas sobre qué es la intervención y sobre sus implicaciones donde reside el carácter perverso de esta práctica en Brasil.

Pude constatar en entrevistas realizadas que el médico pue- de también erigirse como árbitro de la decisión de realizar o no la cirugía esterilizadora, de acuerdo con criterios absolutamente personales.

La exigencia en algunas empresas del comprobante de esterilización como condición para admitir mujeres en su plantilla de empleados ha contribuido a reforzar la "cultura de la esterilización". Además de las tradicionales discriminaciones que las mujeres sufren en el mercado de trabajo y en el ejercicio profesional, más recientemente ha aparecido esta exigencia, condicionando la posibilidad de la mujer de obtener un empleo a su esterilización previa.

En este contexto, se torna bastante problemático afirmar que la realización de la cirugía de esterilización sea un acto de libertad, consciente y voluntario, sin constreñimientos.

La difusión de esta práctica y el fortalecimiento de la "cultura de la esterilización" en períodos más recientes se evidencia cuando se verifica que el $54,7 \%$ de las esterilizaciones fueron hechas en los 5 años inmediatamente anteriores a la encuesta del PNAD (1980-1985), y que en el año de realización de la misma, 1986, habían sido esterilizadas el 9,1\% de las mujeres que se sometieron a este procedimiento. Las restantes, en proporción del $35,7 \%$ fueron esterilizadas antes de 1980, siéndolo un 20,9\% entre 1975 y 1979 y un $14,8 \%$ antes de 1975 . Por estos datos se ve que la tendencia fue, hasta 1986, de aumento del número de mujeres esterilizadas. Investigaciones más recientes, aunque no tengan la extensión de la PNAD, muestran que el cuadro revelado en 1986 no se ha modificado (Berquó, 1993)

La gran mayoría de las intervenciones fue realizada durante el último parto $(75,1 \%)$. Este procedimiento ha contribuído de modo importante en la incidencia de altas tasas de cesáreas que ocurren en el país: en 1980, ya era del 31\% entre las pacientes de la previsión pública, y desde entonces esta cifra sólo ha aumentado. En el Estado de Golás, en 1991, se registró la exhorbitante tasa de 68,7\% de partos quirúrgicos (Berquó, 1993). La mayor parte de los arreglos entre mujeres que quieren ser esterilizadas y los profesionales de salud, revelados en una investigación realizada en Sao Paulo (citada por Berquó, 1993), son hechas durante el embarazo, en diferentes momentos del mismo, incidiendo fuertemente el momento del parto como ocasión propicia para la esterilización. "El 32\% de las mujeres declaran que se embarazaron para ser esterilizadas durante el parto -por cesárea, está claro.” (Berquó, 1993: 376)

\section{CONSIDERACIONES FINALES}

La caída de la fecundidad de las mujeres brasileñas se da en el contexto de la transición hacia una sociedad moderna, urbana e industrial, repitiendo la tendencia ocurrida en los países desarrollados, desde el advenimiento de la industrialización. 
Sin embargo es preciso tomar en cuenta nuestras especificidades históricas en este proceso. La mejoría de incontables indicadores sociales, como la alfabetización, mortalidad general e infantil, y la expectativa de vida, entre otros, resultan no de un proceso de distribución de la riqueza generada por el desarrollo y de la elevación del nivel de la calidad de vida de la mayoría de la población, sino de la expansión de la infraestructura de servicios básicos, como sanidad y atención a la salud, y de la implementación de programas preventivos de largo alcance como, por ejemplo, campañas de vacunación masiva. La gran concentración de la renta y el carácter excluyente del desarrollo brasileño reproducen y hasta profundizan las grandes desigualdades existentes entre las regiones, entre los sectores y entre los segmentos sociales. El contexto político, durante un largo período marcado por el autoritarismo, hizo inviable la participación popular en la reversión de este cuadro y obstaculizó la construcción de una ciudadanía efectiva. En la etapa post-régimen no hubo reducción de las desigualdades económicas y sociales; al contrario, se han acentuado en el contexto de las políticas neo-liberales, así como han sido pocos los avances en la construcción de la ciudadanía deseada.

Esta realidad se expresa también en lo que se refiere a la regulación de la fecundidad. Los factores anteriormente señalados contribuyen a la generalización de actitudes favorables a esta regulación, pero su efectividad no traduce logros significativos para la mayoría de las mujeres, en términos de ciudadanía reproductiva, entendiendo ésta como la posibilidad de participación de las mujeres en la formulación de programas y estrategias de planificación familiar que les garanticen, de hecho, acceso y opciones de procedimientos anticonceptivos, conscientes y adecuados a las características específicas de cada usuaria, permitiendo la vivencia de su sexualidad sin temores y $\sin$ riesgos. En términos institucionales y de legislación, fueron conquistados avances a lo largo de los años 80, como la creación del Consejo Nacional de los Derechos de la Mujer y sus equivalentes estatales y la formulación del Programa de Asistencia Integral de Salud de la Mujer (PAISM), pero su implementación está lejos de cumplir lo previsto. (Berquó, 1993; Ávila, 1993).

Hemos visto que en Brasil la "opción" de regulación de la fecundidad está restringida a dos métodos: los anticonceptivos orales y la esterilización, ambos practicados de modo indiscriminado. El aborto no es computado como un procedimiento regulador de los nacimientos aunque, según estimaciones, sea extensamente utilizado. Por ser ilegal y realizado clandestinamente, no permite la obtención de datos precisos. Su criminalización encubre y facilita la generalización de las condiciones de absoluta falta de seguridad en que es practicado, penalizando, en especial, a las mujeres de estratos socio-económicos bajos, sin recursos para recurrir a clínicas donde, aunque clandestino, el aborto es realizado con mayor seguridad. Esta posibilidad está restringida a mujeres que pueden pagar un alto precio por la intervención y realizarla con menores riesgos.

Este cuadro revela que la caída de la fecundidad en la población brasileña no está siendo acompañada por la constitución de derechos reproductivos. Con la inexistencia real de opciones, la regulación de la fecundidad queda restringida al uso de la píldora y frente a los inconvenientes derivados de los efectos colaterales que a veces provoca, la alternativa casi exclusiva construida y practicada es la esterilización quirúrgica.

El díficil proceso de construcción de la ciudadanía en el país, aún inacabado incluso con el cierre del ciclo autoritario de los gobiernos militares, tienen aún un largo camino por recorrer y precisa incorporar entre sus metas la conquista efectiva de los derechos reproductivos, sin los que sólo se construirá una ciudadanía de segundo orden. Este es uno de los grandes desafíos que al que se enfrenta la población brasileña y, en especial, nosotras las mujeres.

\section{BIBLIOGRAFÍA}

Ávila, Maria Bethánia (1993). "Modernidade e cidadanía reprodutiva". Estudos Feministas. Rio de Janeiro: CIE/ECO/UFRJ, V.I, no 2 (382:383).

Barbosa, Regina M. e Arilha, Margareth (1993): “A experiência como o Cytotec". Estudos Feministas. Rio de Janeiro: CIE/ECO/UFRJ, V.I, nº 2 (408:417).

Berquó, Elza (1982). “Os corpos silenciados”. Novos Estudos Cebrap. Sao Paulo: Ed..Brasileira de Ciências, V.I., nº 3, julho (46:49).

Berquó, Elza (1993)."Brasil, um caso exemplar - anticoncepçao e parto cirúrgico- à espera de uma aço exemplar". Estudos Feministas. Rio de Janeiro: CIEC/ECO/UFRJ, v.I, n.2 (366:381).

CUT/DESEP (1994). Indicadores DESEP/94. Sao Paulo.

DiEESE (1993). Anuário dos Trabalhadores. Sao Paulo.

FARIAS, Vilmar E. (1989). "Políticas de governo e regulaçao da fecundidade: consequências nao antecipadas e efeitos perversos". Ciências Sociais Hoje. Sao Paulo: ANPOCS.

IBGE (1990): Participaçao Político-Social 1988. v.3. Rio de Janeiro.

IBGE (1991). Anticoncepçao, 1986. V.I. Rio de Janeiro: Departamento de Emprego e Rendimento.

IBGE (1993). O Brasil em números. V.2.

IBGE (1994). Mapa do mercado de trabalho no Brasil. n.I. Rio de Janeiro: Diretoria de Pesquisa.

IBGE (1996). Pesquisa Nacional por Amostra de Domicilios - PNAD. Síntese de indicadores, 1993. Rio de Janeiro: Departamento de Emprego e Rendimento.

Istoé (1993). Maternidade amputada. Sao Paulo: Ed.Três, n.1238, 23 junho (72:73).

KABEER, Nalla (1992). From fertility reduccion to reprodutive choice: gender perspective on family planning. Discussion Paper. n.299. Brighton/IDS.

Oliveira, Jane Souto (1993). "Direitos reprodutivos e políticas descartáveis". Estudos Feministas. Rio de Janeiro: CIEC/ECO/UFRJ, v. I. n.2 (400:407)

SCavone, Lucila, Bretin, Héléne e ThêBaud-Mony, Annie (1994). "Contraconcepçao, controle demográfico e desigualdade sociais: análise comparativa franco-brasileira". Estudos Feministas. Rio de Janeiro: CIEC/ECO/UFRJ, v. 2, nº 2 .

Singer, Paul (1977). Economia política do trabalho. Sao Paulo: Hucitec. Simoes, Celso C.S. e Oliveira, Luiz Antonio P. (1988). Perfil estatístico de crianças emes no Brasil: A situaçao da fecundidae: determinantes gerais e características da transiçao recente. Rio de Janeiro: IBGE, Departamento de Estatísticas e Indicadores Sociais.

TANAKA, Ana Cristina d'Adretta (1994). Perfil de saúde da mulher brasileira. Seminário Preparatório à Conferência Mundial sobre a Mulher, organizado pelo Ministério das Relaçoes Exteriores do Brasil. Porto Alegre: 13 a 15 de junho (mimeo).

Terra, Patrícia (1991). "Esterilizaçao. Uma arma política". Cadernos do Terceiro Mundo. Rio de Janeiro: Ed.Terceiro Mundo, a.XIV, n.141, julho (10:17)

YounG, Kate (1989). Serving two masters. Third world in development. New Delhi: Allied. 


\section{RESUMEN}

La autora analiza cómo el argumento sobre los riesgos de la explosión demográfica esgrimidos hasta épocas muy recientes en Brasil, carecen en la actualidad de fundamento ya que la tasa de fecundidad viene cayendo rápida e intensamente en las últimas décadas. Se centra en estas modificaciones, el contexto en que tienen lugar, los procedimientos anticonceptivos responsables de su declive, su utilización en diferentes segmentos socio-económicos de la población y lo que este proceso puede representar para las mujeres, en términos de conquista de una ciudadanía plena que, para ser tal, debe incorporar los derechos reproductivos.

\section{ABSTRACT}

The author analyzes the argument about the risks of demographic explosion exposed until a few years ago in Brazil and concludes that nowadays these arguments lack of groundwork because the fecundity rate is decreasing quickly and profoundly in the last dcades. There are sttled these modifications, the context in which they take place, the anticonceptive procedures responsible for this decrease, their use for different socioeconomic sectors of population and what this process could mean for women, in terms of conquest a full citizenship which needs to take into account the reproductive rights.

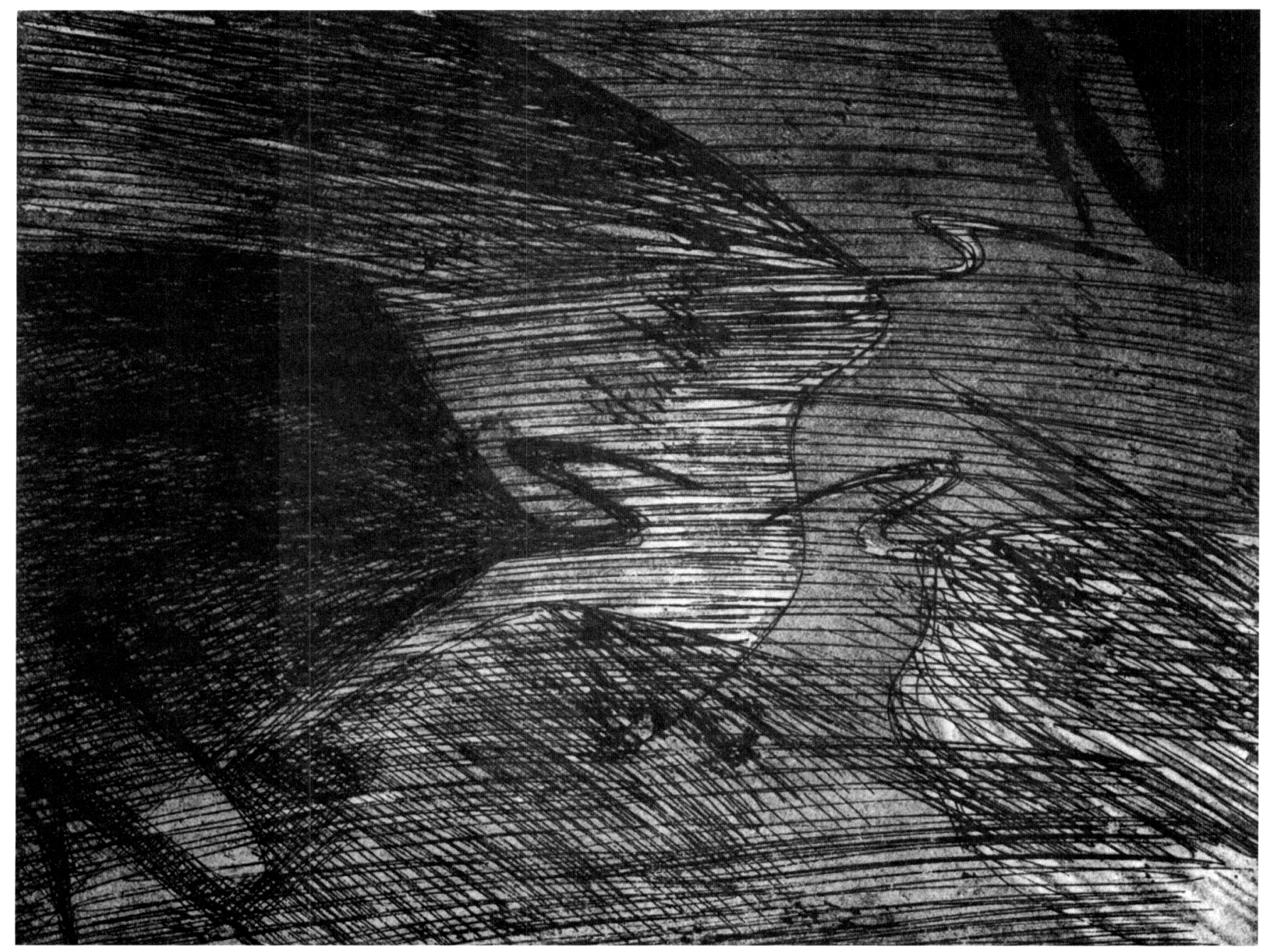

